\title{
Experimental and theoretical study of processes of formation and growth of pearlite colonies in eutectoid steels
}

\author{
V. G. Vaks ${ }^{a, b}$, A. Yu. Stroev ${ }^{a, b}$, V. N. Urtsev $^{c}$ and A. V. Shmakov ${ }^{c}$ \\ ${ }^{a}$ Russian Research Center "Kurchatov Institute", 123182 Moscow, Russia \\ ${ }^{b}$ Moscow Institute of Physics and Technology (State University), 117303 Moscow, Russia \\ ${ }^{c}$ Research-Technological Centre "Ausferr", 455023, Magnitogorsk, Russia
}

(Dated: March 24, 2021)

\begin{abstract}
We describe our optical and electron-microscopy observations of pearlite structures in eutectoid steels which seem to imply that the mechanisms of formation of pearlite colonies in these steels differ from those observed earlier for non-eutectoid steels. A simple theoretical model to study kinetics of pearlite transformations is suggested. Simulations of growth of pearlite colonies based on this model reveal that for the volume carbon diffusion mechanism usually-supposed such growth is always unstable, and the steady-state growth can be realized only via the interfacial carbon diffusion mechanism. A model of formation of pearlite colonies based on the assumption of a strong enhancement of carbon diffusion near grain boundaries is also suggested. The model can be applicable to the plastically deformed steels, and the results of simulations based on this model qualitatively agree with some microstructural features of formation of pearlite colonies observed in such steels.

PACS numbers: $05.70 . \mathrm{Fh} ; 05.10 . \mathrm{Gg}$
\end{abstract}

\section{INTRODUCTION}

Studies of growth and formation of pearlite colonies in steels attract great attention for many decades, see, e. g. [1]-[10] and references therein. However, both the experimental information and the theoretical understanding of these phenomena seem to remain to be rather limited. Most of experimental data used in the literature (see e. g. [1, 2], 7]-[10]) were obtained long ago and by not modern methods, and many important phenomena, in particular, formation of pearlite colonies, seem to be insufficiently studied. In theoretical treatments, only steady-state growth of colonies is usually considered, basing mainly on the equilibrium thermodynamics ideas [3]- [5], but with no attempts of microscopic treatments of kinetic processes. Recently, phenomenological phasefield approaches were used to treat the pearlite growth problem [9, 10]. However, these treatments employ many phenomenological parameters whose physical meaning is not always clear (while no microscopic estimates for them are given), and they discuss only volume but not interfacial diffusion mechanisms (which can be very important for such processes, see [11] and below). Thus the results obtained can hardly significantly elucidate the actual microscopic mechanisms of growth of pearlite colonies.

At the same time, even main mechanisms of pearlite transformations seem to be not well understood as yet. It is unknown whether the colony growth kinetics is determined by the volume or the interfacial diffusion of carbon atoms, and this problem is debated [7, 11]. It is not clear which kinetic or thermodynamic factors determine the colony period $S[1,[8]$. There are absent not only theories but even any definite ideas about the mechanism of formation of colonies near grain boundaries and other lattice defects [1, 2, 8], etc.

This work aims to study some of the problems men- tioned both experimentally and theoretically. In sec. 2 we present some results of our experimental observations of processes of formation of pearlite colonies in an eutectoid steel. These processes are widely discussed in the literature. It is generally believed that the colonies are formed near grain boundaries of austenite or near other defects of crystal lattice [1, 2], but the detailed experimental information about these processes is rather scarce as yet. Most thoroughly they have been discussed by Hillert 2] who studied formation of pearlite colonies in both hypoeutectoid steels (those with the carbon concentration $c$ lower than its eutectoid value $c_{e}$ ) and hypereutectoid steels with $c>c_{e}$. Hillert found that the earliersupposed "repeated sidewise growth" mechanism [1] is not observed in his experiments; instead, "the individual lamellae form by branching during edgewise growth" near various lattice defects: grain boundaries, twinned planes, interphase boundaries, etc. However, the eutectoid steels with $c \simeq c_{e}$ were not studied in Ref. [2]. Therefore, it seems interesting to study the colony formation processes in such steels by the modern methods. In Sec. 2 we describe such studies and compare the results of our observations to those of previous work [1, 2].

The rest part of this work describes some attempts to develop the microscopic approach to the pearlite transformation theory. As a possible first step for that, a simple model of alloys iron-carbon was recently suggested [12]. This is a binary interstitial alloy model with the phase diagrams illustrated by Fig. 5(a) below being symmetric with respect to the interchange of ferrite and cementite. The pearlite growth kinetics for this model was studied basing on the appropriate Ginzburg-Landau functional. The volume diffusion of carbon was supposed to be the main kinetic mechanism of this growth, in accordance with the most of models used in the literature [1]-[5]. The results of simulations of growth of colonies 
made in [12] agreed basically with the conclusions of phenomenological treatments [3]-[5], though some new effects have also been found.

However, the real phase diagram ferrite-cementite (if we model it by a binary alloy model) is by no means symmetric with respect to the interchanges of ferrite and cementite. For example, if we take the carbon concentration in cementite for unity, then the eutectoid concentration is actually close to $c_{e}=1 / 8$, instead of $c_{e}=1 / 2$ in symmetric models. Therefore, to make such approach more realistic, we should study the colony growth also for non-symmetric models with $c_{e} \simeq 1 / 8$. This is done in Secs. 3 and 4 of the present work. We find that for all models of the type considered, the growth of colonies via the volume carbon diffusion mechanism is unstable, and the steady-state growth can be realized only via the interfacial diffusion of carbon. It agrees with the similar conclusion of a recent empirical analysis [7].

In Sec. 5 we suggest a model of formation of pearlite colonies near grain boundaries of austenite. As mentioned, the colonies are usually formed just in this region, but until now there seem to be no microscopic explanations for that [1, 2]. In the model suggested, we relate the formation of colonies to a great enhancement of carbon diffusion near grain boundaries discussed by a number of authors [17, 18]. The results of our treatment show that in the simplest form presented this model can be applied only to the strongly deformed materials, such as those studied in Ref. [6]. However, further generalizations can extend the region of applicability of the approach suggested. Main conclusions are summarized in Sec. 6 .

\section{OBSERVATIONS OF MICROSTRUCTURE OF PEARLITE COLONIES IN NON-DEFORMED AND DEFORMED EUTECTOID STEELS}

\section{A. Preparation of samples and methods of experiments}

All microstructural investigations were made on samples of steel 85 with the composition shown in Table 1. Thus, the concentration of carbon was close to the eutectoid one for alloys iron-carbon: $c_{e}^{\mathrm{Fe}-\mathrm{C}} \simeq 3.45 \%$. It differs the steel used in our study from the hypoeutectoid steels with $c<c_{e}$ and hypereutectoid steels with $c>c_{e}$ investigated by Hillert [2].

Table 1. Composition of samples.

\begin{tabular}{|c|c|c|c|c|c|c|c|c|c|}
\hline Impurity & $\mathrm{C}$ & $\mathrm{Mn}$ & $\mathrm{Si}$ & $\mathrm{Cr}$ & $\mathrm{Ni}$ & $\mathrm{Cu}$ & $\mathrm{S}$ & $\mathrm{P}$ & $\mathrm{Al}$ \\
\hline at. $\%$ & 3.91 & 0.60 & 0.48 & 0.08 & 0.08 & 0.04 & 0.04 & 0.02 & 0.02 \\
\hline
\end{tabular}

Rods of steel 85 of diameter $8 \mathrm{~mm}$ and length about $500 \mathrm{~mm}$ were subjected to various thermal and mechanical treatments described below. These different kinds of treatment are numbered by the same way as figures 1-4 in which we show the microstructure of samples for each kind of treatment.

(1) Heating in a muffled furnace up to $1050^{\circ} \mathrm{C}$, cooling at air up to $650^{\circ} \mathrm{C}$, putting sample in a furnace heated up to $650^{\circ} \mathrm{C}$, annealing at this temperature for $20 \mathrm{sec}$, then quenching in water.

(2) Heating in a muffled furnace up to $1000^{\circ} \mathrm{C}$, cooling in the melt of sodium nitrate $\mathrm{NaNO}_{3}$ at temperature $443^{\circ} \mathrm{C}$ up to reaching the melt temperature, then quenching in water.

(3) Heating in a muffled furnace up to $1005^{\circ} \mathrm{C}$, cooling at air up to $750^{\circ} \mathrm{C}$, plastic deformation at this temperature in a torsional type plastometer with the deformation rate $0.2 / \mathrm{sec}$ up to the strain $23 \%$ (for the surface layer), cooling in the melt of $\mathrm{NaNO}_{3}$ at temperature $443^{\circ} \mathrm{C}$ up to reaching the melt temperature, then quenching in water.

(4) Heating in a muffled furnace up to $1000^{\circ} \mathrm{C}$, cooling in the melt of $\mathrm{NaNO}_{3}$ at temperature $443^{\circ} \mathrm{C}$ up to temperature $750^{\circ} \mathrm{C}$, then quenching from $750^{\circ} \mathrm{C}$ in water.

Samples for microstructural studies were cut in the shape of thin washers parallel to the cross-section of the rod. Then they were subjected to a many-stage grinding and polishing procedure followed by etching in a $5 \%$ solution of nitrogen acid in ethyl alcohol (nital).

For a sample treated according to regime (1), the photograph shown in Fig. 1 has been obtained using the structure analyzer Nikon Epiphot-TME and camera Panasonic WV-CP610/G.

Microstructure of samples treated according to regimes (2)-(4) was studied by the scanning electron microscopy (SEM) methods. Cuts prepared for the optical microscopy studies were used for SEM, too. However, these cuts were subjected to the additional ion etching. It provides a high cleanness of the surface and enables one to reveal fine details of the structure [13]. The high resolution scanning electron microscope "SUPRA 55 " by LEO (ZEISS) with "Gemini" field emission column was used to acquire the images shown in figures $2-4$. The in-lens secondary electron detector combined with a low accelerating voltage $(1.5 \mathrm{keV})$ was found to provide the best quality images. Given that the plates of pearlite intersect the surface of the cut at random angles, no sample tilt was required.

The SEM method was chosen because its high resolution makes it to be particularly suitable for the metallographic studies of alloys with a dispersed structure, such as the eutectoid mixtures treated in this study. The characteristic feature of a topographic contrast in SEM is the enhanced brightness of images of sharp hills and ledges of a surface relief, the so-called "edge-effect" related to the increased output of electrons from such areas 13]. This enhanced brightness is seen in figures $2-4$. The surface of pearlite areas in these figures looks as a welldeveloped relief formed by the combined action of the mechanical loading under polishing and the subsequent etching by nital 2]. At the same time, martensite (obtained from the untransformed austenite) is by about an 
order of magnitude harder than ferrite, and it does not contain cementite inclusions. Therefore, martensite areas at the cut surface are much more flat and uniform than those of ferrite and pearlite, and these martensite areas are seen in figures 4(a) and 4(b) as a grey background.

\section{B. Experimental results}

It is generally accepted that "in the homogeneous austenite, pearlite is formed practically only on grain boundaries" [1]. Our observations agree with this conclusion. In Fig. 1 we show the network of decarburizing formed at the surface of a sample. This network is formed due to the chemical interaction of carbon contained in the steel with the atmosphere oxygen, which results in the formation of gaseous oxides and thus in the removal of carbon from the steel. In Fig. 1, the original grain boundaries of austenite are decorated by chains of tiny bright grains of ferrite. Such decoration arises because the surface decarburizing takes place primarily on the grain boundaries. It is so because, first, the velocity of grain-boundary diffusion of carbon much exceeds that of its diffusion within the grain and, second, because the grain-boundary regions are much easier to access for oxygen as compared to inner parts of grain. Fig. 1 also shows that the positions of these ferrite grains seem to coincide with the probable initial positions of pearlite colonies (seen as dark areas), thus the colonies seem to spread just from these chains. This observation agrees with the above-mentioned conclusion that formation of pearlite colonies takes place on the original grain boundaries of austenite.

Fig. 2 shows the microstructure of a classical tinydispersed plate-like pearlite, or sorbite, for which interplate distances, or colony periods $S$, are about $100-200$ $\mathrm{nm}$. The well-developed relief consisting of relatively thin cementite plates in the ferrite matrix is formed due to the many-stage surface treatment (polishing and etching) described in Sec. 2.1 which removes the surface layer of ferrite but make little effect on cementite. Therefore, dark strips ("cavities") in Fig. 2 correspond to the original (and removed) ferrite, while bright areas ("ridges" of different tilt to the cut plane), correspond to cementite.

It seems natural to suggest that the broad approximately vertical region of ferrite in the middle of Fig. 2, from one side, and the thin layer of cementite along the left edge of this region together with a lot of approximately horizontal colonies adjacent to this layer, from other side, correspond to two opposite sides of an original grain boundary of austenite. Then the enrichment of this left region by carbon (being necessary, in particular, to form the boundary cementite layer mentioned) is naturally explained by "releasing" this carbon from ferrite regions formed to the right of this grain boundary. Therefore, it seems most probable that the abovementioned approximately horizontal colonies to the left of this boundary have been formed just on the boundary ce-
FIG. 1: Network of decarburizing along original grain boundaries of austenite observed for a sample prepared as described in point (1) of Sec. 2.1. Dark areas correspond to pearlite, bright inclusions within these region, to ferrite grains, and the grey matrix, to martensite formed after a quench of an untransformed austenite.

FIG. 2: Microphotograph of a nondeformed sample prepared as described in point (2) of Sec. 2.1.

mentite layer (or near it). At the same time, for pearlite colonies to the right of this boundary, it seems natural to suggest that they have been formed from the ferrite layer. Therefore, Fig. 2 can illustrate both two earlierdiscussed mechanisms of formation of pearlite colonies [1, 2], that from the primary cementite and that from the primary ferrite, as well as the probable close interrelation of these two mechanisms in the eutectoid steel under consideration.

One can also observe in Fig. 2 a number of "defect" lines (that is, cuts of appropriate surfaces) where different colonies seem to meet each other or overcome some obstacles. Most of these lines seem to correspond just to "collisions" of colonies growing from different sides of a grain, but some of them can also correspond to lattice defects, for example, to twin boundaries or subgrains of original austenite.

In the plastically deformed samples, both the transformation kinetics and microstructure change considerably. The transformation rate significantly increases. In our experiments, after the hot treatment described in point (3) of Sec. 2.1, the full transformation time for a cylindric sample of $8 \mathrm{~mm}$ length decreased from 10 to 4 seconds. The degree of order and parallelism of pearlite colonies are notably reduced, and many colonies are fragmented, which is illustrated by frames 3(a)-3(c). However, the main microstructural features of formation of colonies discussed above seem to be present in these samples, too. For example, in frame 3(a) one can distinctly see the cementite layer separating ferrite and plate-like pearlite regions, and this layer (together with its vicinity) again seems to be the most probable place of formation of plate-like pearlite colonies. Analogous places of a probable formation of plate-like pearlite colonies (being, though, less regular) are seen in frames 3(b) and $3(\mathrm{c})$. At the same time, on "ferrite" sides of original grain boundaries, the formation of plate-like pearlite colonies in deformed samples seems to be hampered: according to frames 3(a)-3(c), cementite precipitates in this regions have usually an irregular shape being more close to the globular than to the plate-like one.

FIG. 3: Microphotograph of a deformed sample prepared as described in point (3) of Sec. 2.1. Frames (a), (b) and (c) correspond to different areas of a sample. 
FIG. 4: Microphotograph of a sample prepared as described in point (4) of Sec. 2.1. Frames (a) and (b) correspond to different areas of a sample.

In one of experiments with non-deformed samples, varying cooling and temperature conditions, we succeeded to fix an initial stage of pearlite transformation. Microstructures observed in these experiments are illustrated by Fig. 4 where we see some transformed regions surrounded by the region of martensite (grey colored) formed under a quench of an untransformed austenite. More dark areas again correspond to the original ferrite removed by the polishing and etching treatments mentioned. In frame 4(a), in the part of the transformation volume adjacent to its upper boundary, we seem to observe both formation of plate-like pearlite colonies "from ferrite" (via the mechanism similar to that shown in Fig. 2 to the right of the original grain boundary), and nonplate-like, "globular-type" precipitates of cementite similar to those seen in Fig. 3. At the same time, near right boundaries of the transformed region we see rather regular plate-like colonies (tilted to the cut plane) which could be formed via the mechanism "from the boundary cementite layer" similar to that discussed for the left part of Fig. 2. In frame 4(b), analogous rather regular colonies are observed near lower right boundaries of the transformed region, thus vicinities of these boundaries can be the regions of formation of pearlite colonies, too.

\section{Discussion of experimental results}

The above-described observations enable us to make following conclusions and conjectures about mechanisms of formation of pearlite colonies.

1. These observations agree with the point of view generally accepted [1] that in the homogeneous austenite, the plate-like pearlite colonies form mainly near its grain boundaries.

2. At the same time, our observations seem to imply that the mechanism of this formation is complex and includes at least two different stages. The first stage seems to correspond to the decomposition of austenite into ferrite and cementite within some immediate vicinity of the grain boundary accompanied by a transfer of carbon through this boundary. On the side of this boundary enhanced by carbon, the above-mentioned cementite layer is usually formed, and the ferrite layer is formed on the opposite side of this grain boundary.

3. Then the plate-like colonies start to form, being usually approximately normal to the original grain boundary. The mechanisms of this formation are not clear as yet. However, these mechanisms seem to be certainly different for the "cementite" and "ferrite" sides of this boundary, that is, for the regions enriched and depleted in carbon. It seems to follow, in particular, from a quite different morphology of new-formed colonies in these two regions illustrated by Fig. 2. These differences become still more evident for the plastically deformed samples when the plate-like structure of pearlite colonies usually remains only on the "cementite" side of the boundary, while on its "ferrite" side, precipitates of cementite have often an approximately globular rather than the platelike structure.

4. The above-mentioned conclusions about the "manystep" character of formation of pearlite colonies agree qualitatively with the similar conclusions made by Hillert [2]. However, he studied the hypoeutectoid and hypereutectoid steels rather than the eutectoid ones considered in the present work. In addition to that, Hillert studied mainly processes of formation of pearlite due to the interaction of initial lamellas of ferrite or cementite with various lattice defects, such as twin boundaries, grain boundaries, cementite layers on grain boundaries, etc. The main mechanism of formation of colonies observed in his studies was "branching" of this initial lamellas. For the formation of colonies near grain boundaries of the homogeneous eutectoid austenite studied in our work, such branchings have not been observed. Therefore, one can believe that these branchings are characteristic of formation of colonies in more complex conditions considered by Hillert 2] while we observe more simple processes.

\section{MICROSCOPIC MODELS FOR DESCRIPTION OF PEARLITE TRANSFORMATIONS}

As mentioned, even main mechanisms of pearlite transformation seem to be not quite clear as yet. Therefore, in theoretical approaches to this problem it seems desirable to use only simplest models which yield a reasonable description of phase equilibria between austenite, ferrite and cementite, while for the rest include as small number of model parameters with a clear physical meaning as possible. In the previous work [12], such model has been suggested to study the colony growth in alloys with symmetrical phase diagrams of the type shown in Fig. $5(\mathrm{a})$. Below we generalize this model to the case of more realistic, non-symmetrical phase diagrams.

\section{A. Thermodynamic model}

We consider the interstitial alloys $\mathrm{MeX}_{c}$ in which the number of interstices (interstitial voids) for atoms $\mathrm{X}$ is equal to the number of metallic atoms $\mathrm{Me}$, as in austenite and in simplified models of cementite 14]. Then the concentration $x$ of interstitial atoms $\mathrm{X}$ is related to the average filling of interstices $c$ by the relation: $c=x /(1-x)$, but for brevity, the term "concentration" will be used below for the average filling of interstices, $c$. We consider the models with equilibrium phase diagrams temperature $T$ - concentration $c$, shown in Fig. 5, in which phases $A, D$ and $B$ are analogues of ferrite, austen- 
ite and cementite, respectively. Phase $D$ is treated as a disordered solid solution of interstitial (carbon) atoms in the FCC lattice described by the mean-field ("regular solution") approximation, while the phases $A$ and $B$ are described by the order parameters $\eta \quad \zeta$ which for the uniform equilibrium phases have the following values:

$$
\begin{aligned}
& A:(\eta=1, \zeta=0), \quad B:(\eta=0, \zeta=1), \\
& D:(\eta=0, \zeta=0) .
\end{aligned}
$$

For the iron-carbon alloys, the parameter $\eta$ can be considered to be proportional to the Bain or KurdyumovZaks deformation realizing the FCC-BCC transformation, and $\zeta$ is an analogous parameter describing the transition from austenite to cementite [14].

The inhomogeneous alloy states under consideration are described using the generalized Ginzburg-Landau functional $F$ [15, 20] supposing that the characteristic inhomogeneity lengths $l_{\text {inh }}$ in the $c(\mathbf{r}), \eta(\mathbf{r})$ and $\zeta(\mathbf{r})$ coordinate dependences much exceed the lattice constant $a$ :

$$
F=\int \frac{d^{3} r}{v_{a}}[f(c, \eta, \zeta)+G(\nabla c, \nabla \eta, \nabla \zeta)]
$$

Here $v_{a}$ is volume per iron atom, and $f(c, \eta, \zeta)$ is the free energy of a uniform alloy per iron atom. The gradient term $G$ is a bilinear form of gradients $\nabla c, \nabla \eta$ and $\nabla \zeta$ supposed for simplicity to be isotropic:

$$
\begin{aligned}
G= & g_{c c} \nabla c^{2}+g_{\eta \eta} \nabla \eta^{2}+g_{\zeta \zeta} \nabla \zeta^{2} \\
& +2 g_{c \eta} \nabla c \nabla \eta+2 g_{c \zeta} \nabla c \nabla \zeta+2 g_{\eta \zeta} \nabla \eta \nabla \zeta,
\end{aligned}
$$

while coefficients $g_{i j}$ in this form are supposed to be constant.

For simplicity, we also assume that in our model functional (2) the dependence of function $f(c, \eta, \zeta)$ on parameters $\eta$ and $\zeta$ can be described by polynomials with at most the fourth order. Then, for relations (11) for parameters $\eta$ and $\zeta$ in equilibrium homogeneous phases to hold at all temperatures and concentrations, the function $f(c, \eta, \zeta)$ can be taken in the following form (analogous to that proposed in [16] to model the directional solidification processes):

$$
f(c, \eta, \zeta)=\varphi(c)+\Phi_{1}(c, \eta)+\Phi_{2}\left(c^{\prime}, \zeta\right)
$$

where $c^{\prime}=(1-c)$, and $\varphi(c)$ is the free energy per one interstice in a disordered alloy described for simplicity by the mean-field approximation:

$$
\varphi(c)=T\left(c \ln c+c^{\prime} \ln c^{\prime}\right)-V_{0} c c^{\prime} / 2 .
$$

Positive values $V_{0}$ used below correspond to the effective repulsion between interstitial atoms which qualitatively correctly reproduces the type of carbon-carbon interactions in the real austenite [14]). The $\Phi_{1}(c, \eta)$ and
$\Phi_{2}\left(c^{\prime}, \zeta\right)$ functions are taken in the following form:

$$
\begin{aligned}
\Phi_{1}(c, \eta)= & \lambda_{1} c\left(\eta^{2} / 2-\eta^{3} / 3\right) \\
& +A_{1}\left[\tau_{1} \eta^{2} / 2-\left(\tau_{1}+1\right) \eta^{3} / 3+\eta^{4} / 4\right], \\
\Phi_{2}\left(c^{\prime}, \zeta\right)= & \lambda_{2} c^{\prime}\left(\zeta^{2} / 2-\zeta^{3} / 3\right) \\
& +A_{2}\left[\tau_{2} \zeta^{2} / 2-\left(\tau_{2}+1\right) \zeta^{3} / 3+\zeta^{4} / 4\right] .
\end{aligned}
$$

Here $\tau_{1}$ is $T / 2 T_{\mathrm{A}}$ where $T_{\mathrm{A}}$ is the temperature of the A-D phase transition at $c=0 ; \tau_{2}$ is $\mathrm{T} / 2 T_{\mathrm{B}}$ where $T_{\mathrm{B}}$ is the $\mathrm{B}-\mathrm{D}$ phase transition temperature at $c^{\prime}=0$ (i. e., $c=1$ ), and $\lambda_{1}, \lambda_{2}, A_{1}$ and $A_{2}$ are some positive energy parameters.

Values of the structure parameters $\eta$ and $\zeta$ in equilibrium homogeneous phases are found by minimization of functional (4) with respect to $\eta$ and $\zeta$ at the given concentration $c$. The minimization with respect to $\eta$ yields equation $\partial f / \partial \eta=\partial \Phi_{1} / \partial \eta=0$ with the function $\Phi_{1}$ from (6), while the minimization with respect to $\eta$ yields an analogous equation with the derivative of function $\Phi_{2}$ : with respect to $\zeta$ :

$$
\begin{aligned}
& \eta(1-\eta)\left(\tau_{1}-\eta+\lambda_{1} c / A_{1}\right)=0, \\
& \zeta(1-\zeta)\left(\tau_{2}-\zeta+\lambda_{2} c^{\prime} / A_{2}\right)=0 .
\end{aligned}
$$

Choice of physically acceptable solutions of these equations at different $(T, c)$ values will be explained for the equation (7); treatment of Eq. (8) is similar. In Fig. 6 we show the $\eta$-dependence of the $\Phi_{1}$ function in Eq. (6) for two physically different situations:

$$
\begin{aligned}
& 0<\left(\tau_{1}+\lambda_{1} c / A_{1}\right)<0.5, \\
& 0.5<\left(\tau_{1}+\lambda_{1} c / A_{1}\right)<1 .
\end{aligned}
$$

In both cases, the $\Phi_{1}(\eta)$ function has minima at $\eta=0$ and 1 (and a maximum at a certain intermediate value $\left.\eta=\tau+\lambda c / A_{1}\right)$. However, in the case (91), the right minimum is below the left minimum, so that phase A with $\eta=1$ is thermodynamically favorable, while in the case (10), the left minimum is below the right minimum, and phase $\mathrm{D}$ with $\eta=0$ is thermodynamically favorable. The line $\left(\tau_{1}+\lambda_{1} c / A_{1}\right)=0.5$ separating these two regions in the $(c, T)$ plane is shown as a dashed line in the left part of figures $5(\mathrm{a})-5(\mathrm{c})$. It corresponds to the line of phase transitions between phases $\mathrm{A}$ and $\mathrm{D}$ in the absence of phase separation, i. e., at a constant, "frozen" concentration $c$. Similarly, the dashed line in the right part of figures figures $5(\mathrm{a})-5(\mathrm{c})$ shows the line of phase transitions between phases B and D at a "frozen" concentration $c$.

If we take into account possible phase separation, that is, if we minimize the total free energy (2) also with respect to the number of $\mathrm{Me}$ and $\mathrm{X}$ atoms in each phase, we can write total equilibrium equations for any two phases, 1 and 2 , in the well-known form:

$$
\begin{aligned}
& (\partial f / \partial \eta)_{1}=(\partial f / \partial \eta)_{2}=0 ; \quad(\partial f / \partial \zeta)_{1}=(\partial f / \partial \zeta)_{2}=0 ; \\
& (\partial f / \partial c)_{1}=(\partial f / \partial c)_{2}=\mu ; \quad f_{1}-\mu c_{1}=f_{2}-\mu c_{2}
\end{aligned}
$$

where $\mu$ is the chemical potential of carbon with respect to iron [21]. 
FIG. 5: (a) Phase diagram temperature $T$ - concentration $c$ for symmetric model 1 described by Eqs. (12). Phases $A, B$ and $D$ correspond to ferrite, cementite and austenite; solid lines are two-phase equilibrium curves. Left or right dashed line shows the stability limit of phase $\mathrm{D}$ with respect to transition to phase A or phase B at the fixed concentration $c$. Circle shows the $(T, c)$ values for which simulations of growth of colonies have been made. (b) : Same as in (a) but for model 2 described by Eqs. (13). (c) : Same as in (a) but for model 3 described by Eqs. (14).

FIG. 6: Dependence of $\Phi_{1} / A_{1}$ on $\eta$ in Eq. (6), at the temperature and concentration falling in the range determined by inequalities (9) (solid line), and inequalities (10) (dotted line).

Simulations of phase transformations described below were made mainly for three thermodynamic models: for the symmetric model 1 from [12] with the following values of parameters in Eqs. (3)-(6):

$$
\begin{aligned}
& \lambda_{1,2}=A_{1,2}=5 T_{A}, \quad T_{A}=T_{B}, \quad V_{0}=0, \\
& g_{\eta \eta, \zeta \zeta}=a^{2} T_{A}, \quad g_{c c, c \eta, c \zeta, \eta \zeta}=0,
\end{aligned}
$$

and for two non-symmetric models, 2 and 3, with the following parameter values:

$$
\begin{aligned}
& \text { Model 2: } \lambda_{1}=7.5 T_{A}, \quad A_{1}=5 T_{A}, \quad \lambda_{2}=14 T_{A}, \\
& A_{2}=9.49 T_{A}, \quad T_{B}=2 T_{A}, \quad V_{0}=0, \quad g_{c c}=3 a^{2} T_{A}, \\
& g_{\eta \eta}=0.05 a^{2} T_{A}, \quad g_{\zeta \zeta}=0.5 a^{2} T_{A}, \quad g_{c \eta, c \zeta, \eta \zeta}=0 ; \\
& \text { Model 3: } \quad \lambda_{1}=24 T_{A}, \quad A_{1}=8 T_{A}, \\
& \lambda_{2}=23 T_{A}, \quad A_{2}=30 T_{A}, \quad T_{B}=3.8 T_{A} \\
& V_{0}=T_{A}, \quad g_{c c, \eta \eta, \zeta \zeta}=2 T_{A} a^{2} ; \quad g_{c \eta, c \zeta, \eta \zeta}=0
\end{aligned}
$$

where $a$ is the FCC lattice constant. Equilibrium phase diagrams for these models are shown in Fig. 5. Eutectoid values of concentration and temperature, $c_{e}$ and $T_{e}$, for these models are: for model $1, c_{e}=1 / 2, T_{e}=0.43 T_{A}$; for model $2, c_{e}=1 / 3, T_{e}=0.54 T_{A}$; and for model 3 , $c_{e}=1 / 8, T_{e}=0.83 T_{A}$. Note that in the phase diagram for model 3 shown in frame $5(\mathrm{c})$, the left binodal for the phase equilibrium $\mathrm{A}-\mathrm{B}$ coincides with the $c=0$ axis within accuracy of drawing: solubility limits $c_{s}(T)$ are of the order of $10^{-3}$.

\section{B. Kinetic model}

Diffusion of interstitial (carbon) atoms in the course of pearlite transformations is described using the quasiequilibrium kinetic equation method described in Ref. [19]. In the case of weakly inhomogeneous states under consideration, this equation for local concentration $c(\mathbf{r}, t)$ takes the continuum form [20]:

$$
\partial c / \partial t=-\operatorname{divj} ; \quad j_{\alpha}=-c c^{\prime} \sum_{\beta} D_{\alpha \beta} \nabla_{\beta}(\delta F / \delta c) .
$$

Here $\alpha$ and $\beta$ are Cartesian indices, $D_{\alpha \beta}$ is the diffusivity tensor, while function $\delta F / \delta c=\delta F / \delta c(\mathbf{r})$ (having the meaning of the local chemical potential of carbon atoms) is the variational derivative of functional (2) with respect to local concentration $c(\mathbf{r})$ :

$$
\delta F / \delta c(\mathbf{r})=\partial f(c, \eta, \zeta) / \partial c-2\left(g_{c c} \Delta c+g_{c \eta} \Delta \eta+g_{c \zeta} \Delta \zeta\right)
$$

where $\Delta=\nabla^{2}$ is the Laplace operator. In describing the diffusivity $\mathbf{D}$ we take into account not only the usual volume diffusivity $\mathbf{D}_{v}$ but also possible surface contributions $\mathbf{D}_{s}$ which can be important due to the enhanced diffusion of carbon along incoherent interphase boundaries [2, 7, 17]. Therefore, the diffusivity includes both volume and interfacial terms and is written as follows:

$$
D_{\alpha \beta}=D_{v}^{\alpha \beta}+a^{2} \sum_{\gamma \delta} \varepsilon_{\alpha \gamma} \varepsilon_{\beta \delta}\left(D_{s}^{\eta} \nabla_{\gamma} \eta \nabla_{\delta} \eta+D_{s}^{\zeta} \nabla_{\gamma} \zeta \nabla_{\delta} \zeta\right) .
$$

Here the first term describes the volume diffusion (being, generally, different in different phases which in our model can be described by the dependence of $D_{v}^{\alpha \beta}$ on $\eta$ and $\zeta)$. Terms with $D_{s}^{\eta}$ and $D_{s}^{\zeta}$ describe the surface diffusion of carbon atoms along interfaces austenite-ferrite and austenite-cementite, respectively. Below we discuss only growth of plane pearlite lamellas lying within $(y, z)$ plane. For these 2D problems, Cartesian indices $\alpha, \beta, \gamma, \delta$ in the second sum in (17) are $x$ or $y$, while $\varepsilon_{\alpha \beta}=-\varepsilon_{\beta \alpha}$ is the unit antisymmetric tensor with just two non-zero components: $\varepsilon_{x y}=1 \quad \varepsilon_{y x}=(-1)$.

Temporal evolution of structure parameters $\eta$ and $\zeta$ is described by the time-dependent Ginzburg-Landau equations [20] with two phenomenological relaxation parameters, $\gamma_{\eta}$ and $\gamma_{\zeta}$ :

$$
\begin{aligned}
\partial \eta / \partial t= & -\gamma_{\eta} \delta F / \delta-\gamma_{\eta}\left[\partial \Phi_{1}(c, \eta) / \partial \eta \eta(\mathbf{r})\right. \\
& \left.-2\left(g_{c \eta} \Delta c+g_{\eta \eta} \Delta \eta+g_{\eta \zeta} \Delta \zeta\right)\right] ; \\
\partial \zeta / \partial t= & -\gamma_{\zeta} \delta F / \delta \zeta(\mathbf{r})=-\gamma_{\zeta}\left[\partial \Phi_{2}\left(c^{\prime}, \zeta\right) / \partial \zeta\right. \\
& \left.-2\left(g_{c \zeta} \Delta c+g_{\eta \zeta} \Delta \eta+g_{\zeta \zeta} \Delta \zeta\right)\right] .
\end{aligned}
$$

As discussed in detail in Ref. [12], "phonon" relaxation times $\tau_{p h} \sim\left(\gamma_{\eta}^{-1}, \gamma_{\zeta}^{-1}\right)$ in Eqs. (18) describe relaxation of crystal lattice due to the anharmonic interactions of phonons, and they are by many orders of magnitude shorter than the "diffusional" times $\tau_{d} \sim D a^{2}$ that describe relaxation of concentration according to Eq. (15) being realized by the diffusional jumps of carbon atoms between interstices. Therefore, one can expect that in the course of pearlite transformations under study, structure parameters $\eta$ and $\zeta$ adiabatically fast follow the slowly varying distribution of carbon concentration $c(\mathbf{r}, t)$, minimizing the free energy (2) at each given $c(\mathbf{r}, t)$. In particular, if the local concentration $c=c(\mathbf{r}, t)$ obeys inequality (9), the $\eta(\mathbf{r}, t)$ value should be close to unity (that is, the lattice structure should be close to ferrite), while if inequality (10) is obeyed, we should have: $\eta(\mathbf{r}, t) \simeq 0$ (that is, the lattice structure should be close to austenite), and analogously for $\zeta(\mathbf{r}, t)$. 
Deviations from these "uniform" values of $\eta$ and $\zeta$ (i. e., from their values of "zero-order" in non-uniformity) arise only due to the presence of last, gradient terms in Eqs. (18) which in the Ginzburg-Landau approach used are supposed to be small.

In our simulations, we describe this physical picture as follows. The generalized diffusion equation (15) for the concentration $c=c(\mathbf{r}, t)$ is considered as the main one. In the computations, it is replaced by its finitedifference analog and is solved using standard iterative methods. However, after each step of these iterations, the $c(\mathbf{r}, t)$ values obtained are divided into two groups for which either inequality (9) or inequality (10) holds. For points $\mathbf{r}$ corresponding to the first group, we put $\eta(\mathbf{r}, t)=1$, while for $\mathbf{r}$ corresponding to the second group, we put $\eta(\mathbf{r}, t)=0$. The analogous procedure is made for the $\zeta(\mathbf{r}, t)$ values. After that, both parameters $\eta$ and $\zeta$ start to evolve with time according to the "phonon" equations (18) (again replaced by their finitedifference analogs) for the time interval $(t, t+\Delta t)$ at the fixed values $c(\mathbf{r}, t)$. In these computations, we put for definiteness $\gamma_{\zeta}=\gamma_{\eta}$, and the interval $\Delta t \lesssim 0.5 \gamma_{\eta}^{-1}$ was found to be sufficient for the full relaxation of $\eta$ and $\zeta$ to their "quasi-equilibrium" values $\eta[c(\mathbf{r}, t)]$ and $\zeta[c(\mathbf{r}, t)]$. Then these relaxed $\eta$ and $\zeta$ values are put into Eq. (15) as the initial values for the next iteration in $c(\mathbf{r}, t)$, and so on.

\section{SIMULATIONS OF GROWTH OF EUTECTOID PEARLITE COLONIES}

\section{A. Methods of simulations}

Methods of simulations of the steady-state growth were basically the same as those in Ref. [12]. We employed a simulation volume $V_{s}=\left(L_{x} \times L_{y} \times L_{z}\right)$ in the cubic lattice with periodic boundary conditions along $y$ and $z$ axes. The $y$ axis was chosen along the colony growth direction, while the period $L_{z}$ along $z$ axis was taken $a$, so that the growth of lamellar colonies parallel to the $y z$ plane was simulated. The simulation length $L_{x}$ along $x$ axis was taken as a half of the colony period $S: \quad L_{x}=S / 2$, and the mirror boundary conditions along $x$ axis were used both at $x=0$ and at $x=S / 2$. Differential equations (15) and (18) were replaced by their finitedifference analogs with the space-step $L_{s}$ and the timestep $t_{s}$, and these equations were solved by the standard Runge-Kutta method. Values $L_{s}=a$ and $t_{s}=10^{-3} \tau_{d}$ were usually employed where $\tau_{d}$ has the meaning of a characteristic time of diffusional jumps on the distance $a$, while the relations of this $\tau_{d}$ to diffusivities $D_{v}$ or $D_{s}$ in Eq. (17) are indicated below. The initial distribution of parameters $c(\mathbf{r}), \eta(\mathbf{r})$ and $\zeta(\mathbf{r})$ (illustrated by frames $7 \mathrm{a}, 8 \mathrm{a}$ and 11a) was chosen close to that expected for the steady-state growth as described in detail in Ref. [12].

Our simulations were usually performed for relatively low temperatures: $T \lesssim 0.5 T_{e}$, while actually pearlite
FIG. 7: Evolution of eutectoid colonies via the volume diffusion mechanism for the symmetric model 1 with the phase diagram shown in Fig. 5(a) at temperature $T=0.25 T_{A}$, the period $S=32 a$, and the following values of the reduced time $t^{\prime}=t / \tau_{d}$ : (a) 0 , (b) 50, and (c) 400. The grey level linearly varies with local concentration $c(\mathbf{r})$ between 0 and 1 from white to black.

FIG. 8: Same as in Fig. 3 but at $S=64 a$.

transformations are realized at higher $T \gtrsim 0.9 T_{e}$. However, the colony periods $S$ for such "realistic" $T$ become rather large: $S \gtrsim 500 a$, and simulations of evolution of colonies for such $S$ become time-consuming. At the same time, the main aim of this work is elucidation of just main mechanisms but not quantitative details of transformations, while qualitative manifestations of these mechanisms seem to be weakly sensitive to the temperature $T$ values. In addition to that, in studies of the most important problems, such as the instability of growth of pearlite colonies via the volume diffusion mechanism discussed in Sec. IVB we also made checking simulations at higher $T \sim 0.8 T_{e}$, and the results did not significantly change. Therefore, the main results and conclusions presented below seem to be realistic and reliable in spite of all simplifications in both models and simulation parameters used.

\section{B. Growth of colonies via the volume diffusion of carbon mechanism}

In Figs. 7-10 we present some results of our simulations of growth of eutectoid colonies (i. e. growth of colonies into austenite with the carbon concentration $c_{a}$ equal to the eutectoid one, $c_{e}$ ) via the volume diffusion of carbon mechanism. For these simulations we suppose that the surface diffusivities in Eq. (17) are absent: $D_{s}^{\eta}=D_{s}^{\zeta}=0$, while the volume diffusivity is isotropic: $D_{v}^{\alpha \beta}=D_{v} \delta_{\alpha \beta}$ and it has the same value $D_{v}=a^{2} / \tau_{d}$ in all three phases under consideration, austenite, ferrite and cementite. Various thermodynamic models used for these simulations differ mainly by the eutectoid concentration $c_{e}$ value. Figs. 7 and 8 correspond to the symmetrical model 1 with $c_{e}=1 / 2$, and the phase diagram shown in Fig. 5(a). Fig. 9 corresponds to a "not strongly" asymmetric model 2 with $c_{e}=1 / 3$ and the phase diagram shown in Fig. 5(b). Finally, in Fig. 10 we show results for the more realistic, strongly asymmetric model 3 with $c_{e}=1 / 8$.

The results presented in Figs. 7 and 8 (as well as other results for the growth of colonies in symmetrical models discussed in detail in Ref. [12]) basically agree with conclusions of phenomenological theories [5, 8]. At the same time, Fig. 9 shows that for asymmetric models, this growth becomes not quite stationary: it is accompanied by some oscillations in the colony front structure, where 
FIG. 9: Same as in Fig. 7 but for the weakly asymmetric model 2 with the phase diagram shown in Fig. $5(\mathrm{~b})$ at temperature $T=0.2 T_{A}$, period $S=60 a$, and the following $t^{\prime}$ : (a) 100, (b) 300, (c) 500, and (d) 1000.

FIG. 10: Same as in Fig. 7 but for model 3 with the phase diagram shown in Fig. 5(c) at temperature $T=0.4 T_{A}$, period $S=48 a$, and following $t^{\prime}$ : (a) 3 , (b) 10 , and (c) 12 .

the cementite and ferrite lamellas leave behind each other in turn. However, for a "not strongly" asymmetric model 2 , this non-stationarity seems to be not very important.

However, for strongly asymmetric models, such as our realistic model 3 with $c_{e}=1 / 8$, the analogous instability of the growth leads to the impossibility of formation of a regular structure of pearlite colonies via the volume diffusion mechanism. It is illustrated by Fig. 10 which shows that in this case, already for rather short evolution times: $t \lesssim 10 \tau_{d}$, the ferrite lamellas start to leave behind the cementite lamellas and to fuse with each other. It leads to the isolation ("divorcing") of cementite lamellas from austenite and thus to the locking of their further growth.

The similar locking (which in literature is sometimes called "divorcing of pearlite" [2]) had been observed in our simulations for all models with realistic $c_{e} \simeq 1 / 8$. This phenomenon was found to be insensitive to varying thermodynamic and gradient parameters $\lambda_{i}, A_{i}, T_{A, B}$, $V_{0}$ and $g_{i k}$ in Eqs. (14), as well as temperature $T$, and the diffusivity $D_{v}$ in ferrite and cementite.

Such instability of growth of eutectoid colonies at small $c_{e} \simeq 1 / 8$ seems to be related to the most general relation between the diffusion time $t_{d}$ and the diffusion length $l_{d}$ for the volume diffusion mechanism: $t_{d} \sim l_{d}^{2} / D_{v}$. As the distance $l_{d}$ needed for carbon atoms to diffuse through austenite between centers of the ferrite and the cementite lamellas much exceeds the cementite lamella half-width $l_{c}: l_{d}^{2} \sim 50 l_{c}^{2} \gg l_{c}^{2}$, the diffusion time needed for carbon atoms released from a growing ferrite lamella to reach cementite lamellas much exceeds times of "consumption" of carbon atoms from the space before front of these cementite lamellas. An amount of this fast consumed carbon is much lower than that needed for a noticeable growth of this highly concentrated cementite lamella. Therefore, it grows much slower than adjacent ferrite lamellas whose growth is realized via diffusion of released carbon atoms mainly "forward", into the non-transformed austenite, rather than sideways, to the narrow and low-mobile cementite lamellas. Thus the ferrite transformation front leaves behind the cementite transformation front. Therefore, the steady-state transfer of carbon atoms between ferrite and cementite lamellas (supposed to occur in the phenomenological treatments [3]-[5]) is actually not realized.
FIG. 11: Evolution of eutectoid colonies for model 3 via the interfacial diffusion of carbon mechanism described in the text at temperature $T=0.4 T_{A}$ and different periods $S$. Upper row: $S=44 a$, while the values of $t^{\prime}=t / \tau_{d}$ are: (a) 0 , (b) 500, (c) 1000. Middle row: $S=48 a$, while $t^{\prime}$ are: (d) 100, (e) 400, and (f) 700. Lower row: $S=52 a$, while $t^{\prime}$ are: (g) 100, (h) 500, and (i) 1000.

FIG. 12: Same as in Fig. 11 but at $S=40 a$ and the following $t^{\prime}:$ (a) 50, (b) 100, and (c) 120 .

\section{Growth of colonies via the interfacial diffusion of carbon mechanism}

As the austenite-pearlite interphase boundary is basically incoherent [2], one can expect the surface diffusivity along this boundary to much exceed the volume diffusivity $D_{v}^{a}$ of carbon in austenite, similarly to the grain boundary diffusivity which, according to measurements by Bokshtein et al. [17], exceeds this volume diffusivity by several orders of magnitude. We also note that the volume diffusivity in ferrite at temperatures under consideration exceeds that in austenite by $2-3$ orders of magnitude 22]. Keeping in mind all that and employing also considerations of simplicity, in simulations of growth of colonies via the interfacial diffusion mechanism we supposed the values of effective interfacial diffusivities, proportional to quantities $D_{s}^{\eta}$ and $D_{s}^{\zeta}$ in Eq. (17), and the volume diffusivity in ferrite, $D_{v}^{f}$, to be similar, while the volume diffusivities in austenite and cementite, $D_{v}^{a}$, and $D_{v}^{c}$, to be negligibly small. Therefore, in these simulations we put:

$$
\begin{aligned}
& D_{s}^{\eta}=D_{s}^{\zeta}=20 a^{2} / \tau_{d}, \quad D_{v}^{f}=a^{2} / \tau_{d} \\
& D_{v}^{a}=D_{v}^{c}=0
\end{aligned}
$$

where we also take into account that interphase boundaries in our model have widths $w \sim(4-5) a$, thus the structure parameters gradients $\nabla \eta$ and $\nabla \zeta$ in Eq. (17) can be estimated as: $|\nabla \eta| \sim|\nabla \zeta| \sim(0.2-0.25) / a$.

Some results our simulations for model (19) are presented in Figs. 11-14. Let us discuss these results, First, Fig. 11 shows that the stable steady-state growth of colonies via the interfacial diffusion mechanism is possible, unlike that via the volume diffusion mechanism discussed in Sec. IVB, Fig. 11 also shows that both the growth rate $V$ and the stationary front shape notably vary with the colony period $S$ which qualitatively agrees with conclusions of phenomenological treatments [3]-[5]. At the same time, our microscopic approach reveals many kinetic features which are absent in these treatments. In particular, Figs. 11-14 show that the

FIG. 13: Same as in Fig. 11 but at $S=56 a$ and the following $t^{\prime}:$ (a) 50, (b) 250, and (c) 280 . 
FIG. 14: (A): Ratio of the steady-state colony growth rate $V$ to its maximum value $V_{\max }$ versus the reduced colony period $S / S\left(V_{\max }\right)$. Solid line: our simulations illustrated by Fig. 11 for which we found: $V_{\max }=0.031 a / \tau_{d}$. Dotted and dashed lines: results of phenomenological treatments [4, 5] for the volume and surface diffusion mechanism, respectively. (B): Distribution of colony periods $S$ observed in experiments [1].

growth rate $V$ depends on the period $S$ much stronger than in the phenomenological treatments, and the interval of possible periods $S$ is limited not only from below (by the minimal value $S_{0}$ which, according to Zener [3] , is determined by the balance between the volume gain and the surface loss of free energy under colony growth), but also from above, by some maximum value $S_{\max }$ related to the development at $S>S_{\max }$ of the pearlite divorcing processes illustrated by Fig. 13; these processes are analogous to those shown in Fig. 10.

The resulting dependence $V(S)$ (shown in Fig. 14(A) by solid line) turns out to be much sharper than the analogous phenomenological dependences (shown in Fig. 14(A) by dotted and dashed line) for both the volume and the surface diffusion mechanism. At the same time, this more sharp dependence $V(S)$ seems to better agree with the distribution of periods $S$ observed in experiments and illustrated by Fig. 14(B).

Let us also note that our main conclusion that the growth of pearlite colonies is determined by the surface rather than volume diffusion mechanism agrees with a similar conclusion made by Whiting [7] basing on his analysis of experimental data about the pearlite growth velocities.

\section{MODEL OF FORMATION OF PEARLITE COLONIES NEAR GRAIN BOUNDARIES OF AUSTENITE}

As mentioned, possible mechanisms of formation of pearlite colonies are widely discussed in the literature [1, 2, 8]. However, these discussions include usually either detailed phenomenological description of observations of these complex processes [2], or just general considerations about such mechanisms [1, 8], with no attempts of microscopic treatments or modeling of these processes. In this section we discuss a simple theoretical model of formation of pearlite colonies near grain boundaries of austenite based on the assumption of a great enhancement of carbon diffusivity in this region. We show that in the simplest form described below, this model can be applied only to strongly deformed materials but not to the usual materials with relaxed grain boundaries. However, some of results described below can also be useful for understanding of similar processes near relaxed grain boundaries, in particular, those shown in Figs. 2-4 of the present work.
FIG. 15: Formation of colonies for the model described by relations (A)-(C) in the text for the case $h, h_{0} \gg a$ at $c_{b}=c_{e}=0.125$, the simulation length along $x$ axis equal to $L_{x}=512 a$, and the following values of the reduced time $t^{\prime}=t D_{b} / a^{2}$ : (a) 0, (b) 200, (c) 300, (d) 1000 .

FIG. 16: Same as in Fig. 15 but at $c_{b}=0.25, \quad L_{x}=240 a$, and the following $t^{\prime}$ : (a) 0, (b) 50, (c) 100, (d) 300 .

\section{A. Kinetic model}

The thermodynamics of transformation will be described by our most realistic model 3 with parameters given by Eqs. (14). In treatment of kinetics, it is convenient to separately consider the formation of colonies near grain boundaries of austenite and their subsequent growth inside the grain. For brevity, these two stages of evolution will be referred to as the "formation" and the "growth inside the grain" stages. In simulations of the formation stage we made the following assumptions.

(A) Within a layer of a width $h$ adjacent to a plane grain boundary, the volume diffusivity of carbon much exceeds both volume and surface diffusivities within the grain. In our modeling, we describe it by the following relation generalizing Eq. (17) to this non-uniform case:

$$
\begin{aligned}
& 0<y<h: \quad D_{\alpha \beta}=D_{b} \delta_{\alpha \beta}, \\
& h<y: \quad D_{\alpha \beta}=D_{b} \delta_{\alpha \beta}\{1-\exp [(y-h) / l]\}
\end{aligned}
$$

where parameter $l$ characterizes the width of transition to the inner part of grain.

(B) This boundary layer is enriched by carbon and has concentration $c_{b}>c_{e}$ while the concentration within the grain is $c_{e}$.

(C) At the initial time $t=0$, there exist a plane lamella of cementite (or cementite with adjacent ferrite) which has a length $h_{0}$, width $w_{0}$, and is normal to the grain boundary, as illustrated by frames (a) in Figs. 1519.

These assumptions qualitatively agree with the available experimental observations and theoretical considerations. In particular, Bokshtein et al. [17] found the carbon diffusivity near grain boundaries in ferrite at $T=550^{\circ}$ to exceed that within the grain by $3-4$ orders of magnitude, and they observed a similar (though somewhat lower) enhancement of diffusion near grain boundaries of austenite. Authors of a recent theoretical work [18] discussed acceleration of diffusion near grain boundaries in strongly deformed materials; they concluded that the enhancement of diffusion in such materials should spread for the significant distances from grain boundaries. The enhanced concentration of carbon and carbides in vicinities of grain boundaries was noted by a number of authors [1, 17]. For the processes of formation of colonies discussed above in connection with Figs. 2 and 3, such enhancement seems to occur on the "cementite" sides of grain boundaries, and so on. 
FIG. 17: Same as in Fig. 15 but at $c_{b}=0.25, \quad L_{x}=240 a$, and the following $t^{\prime}$ : (a) 0, (b) 50, (c) 100, and (d) 300 .

FIG. 18: Same as in Fig. 15 but at $c_{b}=0.25, \quad l=4 a$, $w_{0}=6 a, h=h_{0}=20 a, L_{x}=600 a$, and the following $t^{\prime}$ : (a) 0, (b) 100, (c) 500, (d) 1000, (e) 1700, and (f) 2800.

Methods of simulations of formation of colonies for the model (A)-(C) were basically the same as those described in Sec. 4. Differences concern only boundary conditions. For "one-dimensional" simulations shown in Figs. 15 and 16 , we put no boundary conditions along $x$ axis, while for the simulations shown in Figs. 17-20, the mirror symmetry with respect to both plane $x=0$ and plane $y=0$ was supposed.

After a relatively fast formation of a colony near grain boundary discussed above, its further growth into the grain was supposed to occur via the interfacial diffusion mechanism described in Sec. 4.3. To simulate this process, we used the following model.

(D) The initial carbon concentration $c(\mathbf{r}, t=0)=c_{0}(y)$ starts to gradually decrease to its volume value $c_{e}$ when the distance $y$ from the grain boundary exceeds the width $y_{0}$ of the layer enhanced by carbon:

$$
\begin{aligned}
& y<y_{0}: c_{0}=c_{b} \\
& y>y_{0}: c_{0}=c_{e}+\left(c_{b}-c_{e}\right) \exp \left[\left(y-y_{0}\right) / l_{c}\right]
\end{aligned}
$$

In our simulations we used such values of parameters: $c_{b}=0.25, c_{e}=0.125, y_{0}=40 a, l_{c}=10 a$.

(E) Diffusivity for this process corresponds to the interfacial diffusion mechanism described by Eqs. (17) and (19).

(F) As the initial state for this modeling, we used the distribution of parameters $c(\mathbf{r}), \eta(\mathbf{r})$ and $\zeta(\mathbf{r})$ obtained in the end of simulation of formation of colonies for $h=h_{0}=13 a$ shown in Fig. 19. This distribution is presented in frame $19(\mathrm{~d})$ for the interval of $x$ between center of the second cementite lamella (nearest to the initial one) and center of the third ferrite lamella, with mirror boundary conditions with respect to both boundaries of this interval along $x$-axis.

\section{B. Results of simulations of processes of formation of pearlite colonies}

Some results of simulations based on the abovedescribed models are shown in Figs. 15-21. Let us first discuss the results presented in Figs. 15 and 16 which correspond to a limiting case of a "very thick" layer

FIG. 19: Same as in Fig. 17 but at $h=h_{0}=15 a, L_{x}=480 a$, and the following $t^{\prime}$ : (a) 0, (b) 100, (c) 500, (d) 1000, (e) 1700, and (f) 2300 .
FIG. 20: Same as in Fig. 17 but at $h=h_{0}=13 a, L_{x}=300 a$ and the following $t^{\prime}$ : (a) 0, (b) 100, (c) 1000, and (d) 1600 .

FIG. 21: Same as in Fig. 17 but at $h=h_{0}=10 a, L_{x}=192 a$, and the following $t^{\prime}$ : (a) 100, (b) 500, and (c) 1000 .

of enhanced diffusion: $h, h_{0} \gg a$, when the evolution becomes effectively one-dimensional. Our simulations showed that in this case, the perfectly periodic pearlite structures are formed practically at any width of initial lamellas $w_{0}$ and any concentration $c_{b}$ within the layer, as for this geometry there are no "losses" of carbon atoms from the phase transformation zone. For example, when a ferrite lamella grows into austenite, "excess" carbon atoms in austenite released from ferrite are accumulated before the front of this lamella until their local concentration exceeds the "critical" value that corresponds to the right dashed curve in the phase diagram of Fig. 5(c) (for the given temperature, $T$ ). Then a cementite lamella starts to form via the "adiabatic" mechanism described in Sec. 3.2. Later on this lamella thickens consuming the carbon atoms before its front, and so on. Note that when concentration $c_{b}$ in the layer is equal to the eutectoid one, $c_{b}=c_{e}$, the period of a self-organized colony obtained in the simulation shown in Fig. 15: $S_{b} \simeq 39 a$, is very close to the minimal period of the steady-state growth shown in Fig. 14(A): $S_{0} \simeq 40 a$.

Figs. 17-20 illustrate evolution of microstructure for more realistic cases of a finite width $h$ of a layer of enhanced diffusion. We found that the type of this evolution is weakly sensitive to varying parameters $l, w_{0}$ and $h_{0}$ mentioned in points $(\mathrm{A})$ and $(\mathrm{C})$, but it sharply depends on the width $h$ of the layer of enhanced diffusion. In particular, at $h=20 a$ (Fig. 17), in the pearlite colony formed via this mechanism survive only cementite lamellas formed "in one", see frame 17(f). Values $h \sim(13-15) a$ (Figs. 18 and 19) seem to be "optimal" for formation of regular colonies, but their period $S$ sharply depends on the value of width $h$ varying from $S \simeq 35 a$ at $h=15 a$, to $S \simeq 47 a$ at $h=13 a$. Finally, in too narrow layers: $h \lesssim 10 a$ (Fig. 20), pearlite colonies do not form. Therefore, formation of regular pearlite colonies via the model mechanism $(\mathrm{A})-(\mathrm{C})$ is possible only if the width of the layer of enhanced diffusion notably exceeds interatomic distances: $h \gtrsim 10 a \gg a$.

If this condition is obeyed and a colony can be formed near grain boundary via the mechanism (A)-(C), its further growth into the grain can be described by the model (D)-(F) of the previous section. To simulate this process we used the above-described formation model with

FIG. 22: Growth of new-formed colonies into the grain for the model described by relations (D)-(F) at $L_{x}=73 a \quad L_{y}=73 a$, and the following $t^{\prime}=t / \tau_{d}$ : (a) 100, (b) 700, b (c) 1000, wjere $\tau_{d}$ is the same as in Sec. 4.3. 
FIG. 23: Role of subgrains of austenite in formation of subcolonies of pearlite (interrupted pearlite transformation) in a plastically deformed steel. Symbol "g.b." means grain boundary of austenite, "s.b." means subgrain of austenite, and dark regions correspond mainly to cementite (photo 34 in [6] $)$.

FIG. 24: Formation of lamellas from initial globulae of cementite on a subgrain of austenite (photo 35 in [6]).

$h=13 a$ for which the period of new-formed colonies observed in Fig. 19: $S \simeq 47 a$, is close to that corresponding to the maximum steady-state growth rate and shown in Fig. $14(\mathrm{~A}): S\left(V_{\max }\right)=48 a$. In Fig. 21 we present some results of this simulation. One sees that the colony steadily grows with the rate $V \sim V_{\max }$ which seems to be natural for the model used with $S \simeq S\left(V_{\max }\right)$.

Now let us discuss a possible relation of a simple pearlite formation mechanism described in this section to reality. As mentioned, such mechanism can be effective only if the enhanced diffusion layer is sufficiently wide: $h \gtrsim(13-15) a$. For the usual, relaxed grain boundaries such widths seem to be too high. However, in materials subjected to an intense plastic strain, the enhanced diffusion regions, according to theoretical estimates [18], should significantly broaden, and the inequality mentioned can be realized. In this connection, it can be interesting to compare our figures 18 and 19 to some experimental data about formation of pearlite colonies in strongly deformed steels obtained by Tushinsky et al. [6] and presented in Figs. 22 and 23. Tushinsky et al. believe that these micrographs show formation of pearlite colonies on subgrains of austenite arisen due to the intense "thermoplastic hardening" of this steel. The morphology of some colonies seen in these figures, in particular, those positioned to the left of the center of Fig. 22, seems to be rather similar to that shown in Figs. 18 and 19 .

In the usual, not deformed steels, the pearlite colonies seem to be formed via more complex, many-stage processes discussed in Sec. 2 and Ref. [2]. However, one can believe that the peculiar features of transformations with strongly inhomogeneous and anisotropic diffusivity described above can also be manifested in these, manystage paths of formation of pearlite colonies.

\section{CONCLUSIONS}

Let us summarize the main results of this work.

1. The microstructure of pearlite colonies in both nondeformed and plastically strained eutectoid steels has been investigated using the optical and the scanning electron microscopy methods. The results obtained enable us to make some new conclusions about mechanisms of formation of pearlite colonies, in particular, about their many-step character and about differences of these mechanisms for the processes of formation of colonies near grain boundaries in eutectoid steels and near other lattice defects in non-eutectoid steels.

2. A simple model for theoretical studies of pearlite transformations is proposed. In spite of its simplicity, it seems to reflect the most significant features of thermodynamics and kinetics of phase transformations between austenite, ferrite and cementite.

3. Simulations of growth of pearlite colonies based on this model showed that the volume diffusion of carbon mechanism supposed in the most of existing theories leads to the instability of the steady-state growth of colonies at any parameters of the model and any temperatures. A more adequate theory of growth based on the mechanism of interfacial diffusion of carbon is suggested. The kinetic characteristics of growth obtained in this theory differ notably from those obtained in the standard phenomenological models but they seem to better agree with available experimental data.

4. A model of formation of pearlite colonies based on the assumption of a strong enhancement of carbon diffusion near grain boundaries of austenite has been suggested. The results of simulations of processes of formation of pearlite colonies based on this model seem to qualitatively agree with the available data for plastically deformed steels. Further generalizations of this model can help to understand more complex many-stage processes observed under formation of pearlite colonies in non-deformed steels.

\section{ACKNOWLEDGMENTS}

The authors are much indebted to Profs. M. Hillert and V. V. Popov for critical comments and valuable advices; to M. K. Zaluletdinov, for participation in performing experiments, and to Yu. N. Gornostyrev, V. N. Degtyarev and. P. A. Korzhavy, for numerous valuable discussions. The work was supported by the Russian Fund of Basic Research (grant No. 06-02-16476); by the fund for support of leading scientific schools of Russia (grant No. NS-3004.2008.2); and by the program of Russian university scientific potential development (grant No. 2.1.1/4540).
[1] R. F. Mehl and W. C. Hagel, Progr. in Metal Phys. 6, 74 (1956).
[2] M. Hillert, in: Decomposition of austenite by diffusional 
processes, ed. by V. F. Zackay and H. I. Aaronson (Wiley, N.-Y.-London, 1962), 197-247.

[3] C. Zener, Trans. AIME. 167, 757 (1945).

[4] M. Hillert, Jernkont. Ann. 141, 757 (1957).

[5] M. Hillert, Metall. Trans. 3, 2729 (1972).

[6] L. I. Tushinsky, A. A, Bataev, L. B. Tikhomirova, Structure of pearlite and strength of constructional steels (Nauka, Novosibirsk, 1993) [in Russian].

[7] M. J. Whiting, Scripta Mater. 43, 969 (2000).

[8] V. M. Schastlivtsev, D. A. Mirzaev, I. L. Yakovleva et al., Pearlite in carbon steels (Inst. of Metal Physics, Ekaterinburg, 2006), Chap. 2 [in Russian].

[9] K. Nakajima, M. Apel, I. Steinbach, Acta Mater. 54, 3665 (2006).

[10] I. Steinbach, M. Apel, Acta Mater. 55, 4817 (2007).

[11] L. Amirouchi, M. Plapp, Acta Mater. 57, 237 (2009).

[12] V. G. Vaks, A. Yu. Stroev, JETP 107, 90 (2008).

[13] B. S. Bokshtein, Yu. G. Veksler, B. A, Drozdovsky et al., in: Metallurgy and thermal treatment of steels and cast iron. Vol 1. Methods of studies and testing. Eds. A. G.
Rakhshtadt, L. M. Kaputkina, S. D. Prokoshkin, A. V. Supov (Moscow, Intermet Engeneering, 2004), pp. 396418 [in Russian].

[14] V. G. Vaks, K. Yu. Khromov, JETP 106, 265 (2008).

[15] V. G. Vaks, K. Yu. Khromov, JETP 109, 619 (2009).

[16] M. Plapp and A. Karma, Phys. Rev. E 66, 061608 (2002).

[17] S. Z. Bokshtein et al., Metal Science and Heat Treatment 3, 6 (1961).

[18] A. G. Kesarev, V. V. Kondrat'ev, Materialovedenie, No. 4, 60-64 (2010) [in Russian].

[19] V. G. Vaks, Phys. Rep. 391, 157 (2004).

[20] I. R. Pankratov and V. G. Vaks, Phys. Rev. B 68, 134208 (2003).

[21] V. G. Vaks, I. A. Zhuravlev, K. Yu. Khromov, JETP 111, 796 (2010)

[22] M. E. Blanter, Phase Transformations in the Course of Heat Treatment of Steels (Metallurgizdat, Moscow, 1962), Chap. 1 [in Russian]. 
This figure "fig1.JPG" is available in "JPG" format from: http://arxiv.org/ps/1011.5086v1 
This figure "fig2.JPG" is available in "JPG" format from: http://arxiv.org/ps/1011.5086v1 
This figure "fig3.JPG" is available in "JPG" format from: http://arxiv.org/ps/1011.5086v1 
This figure "fig4.JPG" is available in "JPG" format from: http://arxiv.org/ps/1011.5086v1 
This figure "fig5.JPG" is available in "JPG" format from: http://arxiv.org/ps/1011.5086v1 
This figure "fig6.JPG" is available in "JPG" format from: http://arxiv.org/ps/1011.5086v1 
This figure "fig7.JPG" is available in "JPG" format from: http://arxiv.org/ps/1011.5086v1 
This figure "fig8.JPG" is available in "JPG" format from: http://arxiv.org/ps/1011.5086v1 
This figure "fig9.JPG" is available in "JPG" format from: http://arxiv.org/ps/1011.5086v1 
This figure "fig10.JPG" is available in "JPG" format from: http://arxiv.org/ps/1011.5086v1 
This figure "fig11.JPG" is available in "JPG" format from: http://arxiv.org/ps/1011.5086v1 
This figure "fig12.JPG" is available in "JPG" format from: http://arxiv.org/ps/1011.5086v1 
This figure "fig13.JPG" is available in "JPG" format from: http://arxiv.org/ps/1011.5086v1 
This figure "fig14.JPG" is available in "JPG" format from: http://arxiv.org/ps/1011.5086v1 
This figure "fig15.JPG" is available in "JPG" format from: http://arxiv.org/ps/1011.5086v1 
This figure "fig16.JPG" is available in "JPG" format from: http://arxiv.org/ps/1011.5086v1 
This figure "fig17.JPG" is available in "JPG" format from: http://arxiv.org/ps/1011.5086v1 
This figure "fig18.JPG" is available in "JPG" format from: http://arxiv.org/ps/1011.5086v1 
This figure "fig19.JPG" is available in "JPG" format from: http://arxiv.org/ps/1011.5086v1 
This figure "fig20.JPG" is available in "JPG" format from: http://arxiv.org/ps/1011.5086v1 
This figure "fig21.JPG" is available in "JPG" format from: http://arxiv.org/ps/1011.5086v1 
This figure "fig22.JPG" is available in "JPG" format from: http://arxiv.org/ps/1011.5086v1 
This figure "fig23.JPG" is available in "JPG" format from: http://arxiv.org/ps/1011.5086v1 